\title{
Systematic review of psychotherapy for adults with functional neurological disorder
}

\author{
Myles Gutkin (D) , 1,2 Loyola McLean (D) ,' Richard Brown (D) , 5,6 \\ Richard A Kanaan
}

- Additional material is published online only. To view, please visit the journal online (http://dx.doi.org/10.1136/ jnnp-2019-321926).

1 Department of Psychiatry, The University of Melbourne, Austin Health, Heidelberg, Victoria, Australia

${ }^{2}$ Consultation-Liaison Psychiatry Department, Royal North Shore Hospital, Saint Leonards, New

South Wales, Australia

${ }^{3}$ Brain and Mind Centre,

The University of Sydney, Camperdown, New South

Wales, Australia

${ }^{4}$ Westmead Psychotherapy Program for Complex Traumatic Disorders, Cumberland Hospital, North Paramatta, New South Wales, Australia

${ }^{5}$ Division of Psychology and Mental Health, The University of Manchester, Manchester, UK ${ }^{6}$ Greater Manchester Mental Health NHS Foundation Trust, Manchester, UK

\section{Correspondence to} Dr Myles Gutkin, ConsultationLiaison Psychiatry Department, Royal North Shore Hospital, Saint Leonards NSW 2065, New South Wales, Australia; myles. gutkin@unimelb.edu.au

Received 26 August 2019 Revised 10 September 2020

Accepted 23 September 2020

Published Online First 5

November 2020

Check for updates

(C) Author(s) (or their employer(s)) 2021. No commercial re-use. See rights and permissions. Published by BMJ.

To cite: Gutkin M, McLean L, Brown R, et al. J Neurol Neurosurg Psychiatry 2021:92:36-44.

\section{ABSTRACT}

Functional neurological disorder (FND) is a common and disabling disorder that is often considered difficult to treat, particularly in adults. Psychological therapies are often recommended for FND. Outcome research on psychological therapies for FND has grown in recent years but has not been systematically evaluated since 2005. This study aims to build on that by systematically reviewing the evidence-base for individual outpatient cognitive behavioural and psychodynamic psychotherapies for FND. Medical databases were systematically searched for prospective studies of individual outpatient psychotherapy for FND with at least five adult participants. Studies were assessed for methodological quality using a standardised assessment tool. Results were synthesised, and effect sizes calculated for illustrative purposes. The search strategy identified 131 relevant studies, of which 19 were eligible for inclusion: 12 examining cognitive behavioural therapy (CBT) and 7 investigating psychodynamic therapy (PDT). Eleven were pre-post studies and eight were randomised controlled trials. Most studies recruited a single symptom-based subtype rather than all presentations of FND. Effect sizes, where calculable, showed generally medium-sized benefits for physical symptoms, mental health, well-being, function and resource use for both CBT and PDT. Outcomes were broadly comparable across the two therapy types, although a lack of high-quality controlled trials of PDT is a significant limitation, as is the lack of long-term follow-up data in the majority of identified CBT trials. In conclusion, both CBT and PDT appear to potentially offer some benefit for FND, although better quality studies are needed.

\section{INTRODUCTION}

Functional neurological disorder (FND) is characterised by distressing or disabling neurological symptoms that are inconsistent with other recognised neurological conditions. It is the most common single diagnosis in first presentations to outpatient neurology clinics and the second most common neurological presenting symptom after headache. ${ }^{1}$ It is also associated with high rates of comorbid mental illness and disability. ${ }^{2}$ Although FND presents with many different symptoms, and there may be distinct variants with epidemiological differences (such as psychogenic non-epileptic seizures, PNES), it is generally thought of as a single disorder with a core set of underlying processes. ${ }^{3}$

Contemporary explanations for FND emphasise dissociative and attentional mechanisms as well as neuroplastic changes in emotion processing and agency perception. ${ }^{4}$ Heightened preconscious emotional responsiveness, affective arousal, disrupted affect regulation and altered interoception of bodily emotional responses in people with FND are thought to contribute directly to the generation of FND symptoms, through limbic influences on awareness and control of sensory, motor and behavioural functions, with early or prolonged psychosocial adversity being a common driver of these alterations. ${ }^{5}$ Cognitive (eg, symptom-focused attention) and behavioural (eg, avoidance) factors are also thought to play a key role in maintaining the condition. ${ }^{6}$

Psychological therapy is usually recommended for all forms of FND, with different therapies targeting different factors in the development and maintenance of the disorder. We group the two main types of therapies as psychodynamic therapy (PDT) and cognitive behavioural therapy (CBT).

There are several different 'brands' of PDT that differ in their emphases, but which share a number of characteristic intervention components ${ }^{7}$ that are collectively referred to as PDT here. All aim to connect FND symptoms with emotional and interpersonal conflicts and experiences in the past or present. A key aim in each case is to increase the ability to acknowledge, tolerate and communicate about difficult experiences and to manage associated stressors more effectively. There is a large theoretical literature on the psychodynamic treatment of FND, which can ultimately be traced to its origins in Freudian psychoanalysis and related approaches (eg, Janet). ${ }^{8}$

CBT for FND typically focuses on reducing symptoms by challenging maladaptive beliefs and reversing avoidance and other unhelpful illness behaviours. ${ }^{4}$ An active, empirical approach is adopted, with an emphasis on behaviour change and hypothesis testing. We use the term broadly in this article to refer to all therapies with their theoretical origins in cognitive psychology, including those designed to identify and challenge unhelpful appraisals and behaviours ${ }^{9}$ (so-called 'second wave' CBT) and those targeting specific cognitive processes, such as perseverative cognition or threat monitoring (so-called 'third wave' approaches).

Unlike PDT, CBT focuses on cognitive and behavioural factors that are maintaining distress and disability in the here and now, with relatively little emphasis on emotional and interpersonal processes. The decreased emphasis on the historical origins of 


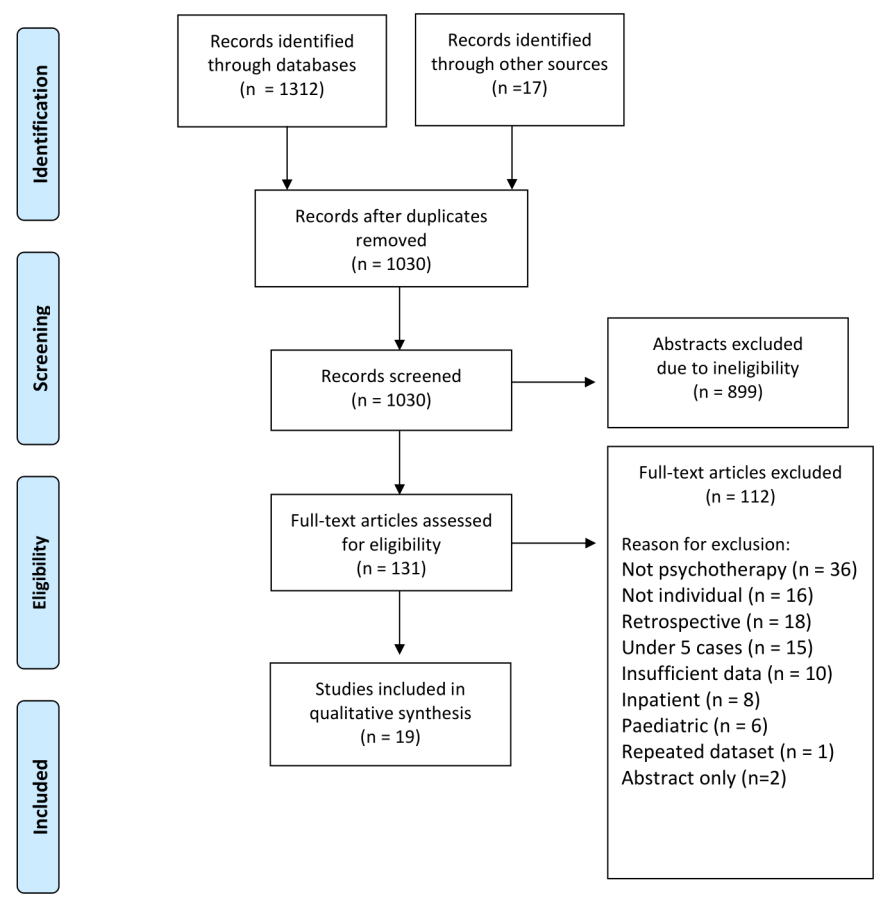

Figure 1 Study selection flowchart.

FND symptoms and the potential role of childhood adversity, which can be sources of conflict and resistance in therapy, ${ }^{10}$ mean that it may be more palatable for some patients than PDT. One potential disadvantage of this emphasis on illness beliefs and behaviours is that potentially important emotional and interpersonal factors may go unrecognised and unaddressed. PDT, in contrast, is particularly suited to addressing these emotional and interpersonal aspects, ${ }^{7}$ although it may not be helpful for patients who are unable or unwilling to examine their problems from this perspective.

A systematic review of psychosocial interventions for FND in $2005^{11}$ concluded that all the available interventions should be viewed as experimental, with only slight evidence in favour of help rather than harm. At that time, only studies of paradoxical intention therapy and hypnosis were eligible for inclusion, neither of which are commonly used in the treatment of FND now. Changes in diagnostic and management approaches since then have made diagnosis and recruitment of patients with FND easier, contributing to an increase in psychological intervention studies. A recent meta-analysis of 16 studies suggests that CBT is potentially beneficial for the subtype of PNES, ${ }^{12}$ with $47 \%$ of eligible participants being seizure free at treatment completion, and $82 \%$ experiencing a reduction in seizure frequency of $50 \%$ or more. Similar analyses have not yet been conducted on CBT treatments for the broader diagnosis of FND, the potential benefits of which are less well understood. Several recent studies have also investigated outcomes following PDT for different types of FND but there has been no published attempt to summarise this literature, meaning that the efficacy of this approach also remains uncertain.

In this paper, we systematically review the evidence for outpatient individual psychotherapy for adults with FND, with a focus on the evidence for PDT and CBT. Primarily psychoeducational, behavioural and hypnotic interventions were excluded in order to focus on the utility of interventions specifically based on psychological theories about the aetiology and treatment of FND.

\section{METHODS}

A systematic search strategy (online supplemental appendix 1) was applied to three major online databases (MEDLINE, PsycINFO and Embase) to identify all prospective treatment studies conducted on adults with any FND subtype with at least five participants in the psychotherapy arm. Treatment had to be psychological therapy administered to individuals in an outpatient setting. Both controlled and uncontrolled studies were included. Outcome variables measured had to include at least one of: physical symptoms, mental health symptoms, quality of life, function, treatment satisfaction and healthcare use. Results were limited to articles in English published from the 1st of January 1980 (the year of publication of Diagnostic and Statistical Manual of Mental Disorders, Third Edition (DSMIII), when the diagnosis of FND was revised) to the 1 st of June 2020. Additional studies were identified from the reference lists of identified studies and reviews. Abstracts were examined for compliance with predetermined inclusion criteria by a single reviewer and full-length articles were obtained where relevance was uncertain from the abstract (figure 1).

All studies that met inclusion criteria were assessed for quality using the National Heart, Lung and Blood Institute study quality assessment tools, standardised quality assessment tools which identify potential sources of bias specific to controlled and uncontrolled intervention studies (available at www.nhlbi. nih.gov/health-topics/study-quality-assessment-tools). The tools consist of 14 (controlled) or 12 (pre-post) items for evaluating potential flaws in study methods or implementation, including sources of bias, confounding, study power, the strength of causality in the association between interventions and outcomes, and other factors. A final quality rating (good, fair or poor) was assigned for each study and reasons for a rating of poor were noted.

Where possible, Cohen's d effect sizes for relevant outcome measures were generated by calculating the mean difference between groups and dividing by the pooled standard deviation. Where possible, baseline measures were compared with endof-treatment measures and to each reported follow-up measure separately. This included the treatment arm of controlled studies. For controlled studies, outcome measures were compared between treatment and control arms at end of treatment and at each follow-up time point. No quantitative synthesis was undertaken due to variability in outcome measures and interventions. The review complied with the 2009 Preferred Reporting Items for Systematic Reviews and Meta-Analyses statement and was registered on the PROSPERO database (registration CRD42018104727).

\section{RESULTS}

We identified 19 relevant studies, ${ }^{13-31}$ the characteristics and full author list of which are outlined in table 1. Elsewhere these studies are referred to by the first author's surname followed by the year of publication. Outcomes were divided into physical symptoms, mental health symptoms, well-being (which includes quality of life and measures of general health), function (which includes global, physical and occupational functional measures) and resource use (which includes medical and other healthcare service use).

Tables 2 and 3 display the effect sizes generated by comparing baseline measures to end-of-treatment measures and to each reported follow-up measure separately for PDT and CBT, respectively. Table 4 shows effect sizes produced by comparing outcome measures between treatment and control arms at end 
Neuropsychiatry

\begin{tabular}{|c|c|c|c|c|c|c|c|}
\hline Author/s and year & Location & Population & Sample size & Study & Intervention & Follow-up & NHLBI quality \\
\hline Goldstein et al $2004^{13}$ & London, UK & PNES & 20 & $B+A$ & $\begin{array}{l}\text { CBT } \\
\text { (FEA) }\end{array}$ & 6 months & Good (9/12) \\
\hline LaFrance et al $2009^{14}$ & Rhode Island, USA & PNES & 21 & $\mathrm{~B}+\mathrm{A}$ & $\begin{array}{l}\text { CBT } \\
\text { (ES-MDD) }\end{array}$ & Treatment end & Good (10/12) \\
\hline Goldstein et al $2010^{15}$ & London, UK & PNES & $\begin{array}{l}33 \mathrm{CBT}+\mathrm{SMC} \\
33 \mathrm{SMC}\end{array}$ & RCT & $\begin{array}{l}\text { CBT } \\
\text { (FEA) }\end{array}$ & 6 months & Good (11/14) \\
\hline LaFrance et al $2014^{16}$ & Rhode Island, USA & PNES & $\begin{array}{l}9 \mathrm{CBT} \\
9 \mathrm{SSRI} \\
10 \mathrm{CBT}+\mathrm{SSRI} \\
10 \mathrm{SC}\end{array}$ & RCT & $\begin{array}{l}\text { CBT } \\
\text { (ES-MDD) }\end{array}$ & Treatment end & Fair (9/14) \\
\hline Dallocchio et al $2016^{17}$ & Verona, Italy & Motor FND & $\begin{array}{l}11 \mathrm{CBT} \\
8 \mathrm{SC} \\
10 \mathrm{CBT}+\mathrm{APA}\end{array}$ & RCT & $\begin{array}{l}\text { CBT } \\
\text { (FEA) }\end{array}$ & Treatment end & Fair (10/14) \\
\hline Baslet et al $2015^{18}$ & Chicago, USA & PNES & 6 & $\mathrm{~B}+\mathrm{A}$ & $\begin{array}{l}\text { CBT } \\
\text { (FEA+MBT) }\end{array}$ & Treatment end & Poor $(6 / 12)$ \\
\hline Sharpe et a/ $2011^{19}$ & $\begin{array}{l}\text { Edinburgh and } \\
\text { Glasgow, UK }\end{array}$ & All FND & $\begin{array}{l}64 \mathrm{CBT} \\
63 \mathrm{SC}\end{array}$ & RCT & $\begin{array}{l}\text { CBT } \\
\text { (GSH) }\end{array}$ & 6 months & Good (12/14) \\
\hline Myers et al $2017^{20}$ & Pennsylvania, USA & PNES + PTSD & 18 & $\mathrm{~B}+\mathrm{A}$ & $\begin{array}{l}\text { CBT } \\
\text { (PE) }\end{array}$ & Treatment end & Fair (9/12) \\
\hline Graham et al $2018^{21}$ & Leeds, UK & All FND & 8 & $\mathrm{~B}+\mathrm{A}$ & $\begin{array}{l}\text { CBT } \\
\text { (ACT) }\end{array}$ & Treatment end & Fair (9/12) \\
\hline Tolchin et al $2019^{22}$ & Boston, USA & PNES & $\begin{array}{l}31 \text { CBT } \\
29 \text { CBT+MI }\end{array}$ & \#RCT & $\begin{array}{l}\mathrm{CBT} \\
(\mathrm{FEA} \pm \mathrm{MI})\end{array}$ & Treatment end & Good (12/14) \\
\hline Baslet et al $2020^{23}$ & Boston, USA & PNES & 49 & $\mathrm{~B}+\mathrm{A}$ & $\begin{array}{l}\text { CBT } \\
\text { (FEA+MBT) }\end{array}$ & Treatment end & Poor (6/12) \\
\hline Goldstein et al $2020^{24}$ & $\begin{array}{l}\text { England, Scotland and } \\
\text { Wales, UK }\end{array}$ & PNES & $\begin{array}{l}186 \mathrm{CBT}+\mathrm{SMC} \\
181 \mathrm{SMC}\end{array}$ & RCT & $\begin{array}{l}\text { CBT } \\
\text { (FEA) }\end{array}$ & 12 months & Good (12/14) \\
\hline Hinson et al $2006^{25}$ & Chicago, USA & Motor FND & 10 & $\mathrm{~B}+\mathrm{A}$ & $\begin{array}{l}\text { PDT } \\
\text { (STDP) }\end{array}$ & Treatment end & Fair (9/12) \\
\hline Reuber et al $2007^{26}$ & Sheffield, UK & All FND & 91 & $\mathrm{~B}+\mathrm{A}$ & $\begin{array}{l}\text { PDT } \\
\text { (PIT) }\end{array}$ & 6 months & Fair (9/12) \\
\hline Mayor et al $2010^{27}$ & Sheffield, UK & PNES & 47 & $\mathrm{~B}+\mathrm{A}$ & $\begin{array}{l}\text { PDT } \\
\text { (PIT) }\end{array}$ & $31-65$ months & Poor (7/12) \\
\hline Kompoliti et al $2014^{28}$ & Chicago, USA & Motor FND & $\begin{array}{l}7 \text { immediate } \\
8 \text { delayed }\end{array}$ & RCT & $\begin{array}{l}\text { PDT } \\
\text { (STDP) }\end{array}$ & 6 months & Poor (8/14) \\
\hline Hubschmid et al $2015^{29}$ & Lausanne, Switzerland & All FND & $\begin{array}{l}11 \text { treatment } \\
12 \text { control }\end{array}$ & RCT & $\begin{array}{l}\text { PDT } \\
\text { (PIT) }\end{array}$ & 12 months & Fair (10/14) \\
\hline Russell et al $2016^{30}$ & Nova Scotia, Canada & PNES & 28 & $\mathrm{~B}+\mathrm{A}$ & $\begin{array}{l}\text { PDT } \\
\text { (STDP) }\end{array}$ & 3 years & Poor (7/12) \\
\hline Santos et al $2014^{31}$ & Sao Paolo, Brazil & PNES & 37 & $\mathrm{~B}+\mathrm{A}$ & $\begin{array}{l}\text { PDT } \\
\text { (PA) }\end{array}$ & Treatment end & Poor (7/12) \\
\hline
\end{tabular}

\#RCT $=$ RCT analysed as $\mathrm{B}+\mathrm{A}$ in this review.

ACT, acceptance and commitment therapy; APA, adjunctive physical activity; $B+A$, before and after study; CBT, cognitive behavioural therapy; ES-MDD, CBT for epilepsy and major depressive disorder; FEA, fear-escape avoidance model; FND, functional neurological disorder; GSH, guided self-help; MBT, mindfulness-based therapy; MI, motivational interviewing; NHLBI, National Heart, Lung and Blood Institute; PA, psychoanalysis; PDT, psychodynamic therapy; PE, prolonged exposure; PIT, psychodynamic interpersonal therapy; PNES, psychogenic non-epileptic seizures; PTSD, post-traumatic stress disorder; RCT, randomised controlled trial; SC, standard care; SMC, standardised medical care; SSRI, selective serotonin reuptake inhibitor; STDP, short-term dynamic psychotherapy.

of treatment and at each follow-up time point in the controlled studies. Where effect sizes provided by the publication were used, or where effect sizes could not be calculated using the standard technique above, this was noted in the table.

The 19 studies identified were divided into those based on CBT and those based on PDT. A description of the different approaches used in each of the studies is provided in online supplemental appendix 2. ${ }^{32-37}$ Where effects were associated with worse outcomes, the effect size was presented in the table as a negative number. Where effect sizes were calculated using an odds ratio, this was indicated with an asterisk (*) after the number, or where this led to a non-numerical result of infinity, this was represented by an asterisk with no number. Where relevant data were provided in the publication but could not be transformed into effect sizes, this was represented as not calculable.

Of the seven studies that investigated PDT, three treated PNES, two treated all FND and two treated motor FND. The majority were small (median $\mathrm{n}=28$ range $=10-91$ ), although two were considerably larger (Reuber 2007 and Mayor 2010; $n=91$ and $n=47$, respectively). All but two (Hinson 2006 and Santos 2014) collected follow-up data for at least 6 months, and three (Mayor 2010, Hubshmid 2015 and Russell 2016) collected long-term follow-up data for a year or more. Russell 2016 was a substudy of PNES as part of a larger trial, and was judged to be of poor quality due to a lack of detail about patient selection and the post-hoc nature of the analysis, including introducing new outcome measures over the course of the trial. Santos 2014 was rated as poor quality due to selection bias from self-selection into the treatment group (11 of 48 refused to participate and were excluded) and a single self-report outcome measure which introduced reporting bias.

Regarding the two larger studies, Reuber 2007 was an uncontrolled practice-based evaluation of a modified form of psychodynamic interpersonal therapy (PIT) treating all FND that reported statistically significant benefits in all domains measured, which were sustained at 6 months. Mayor 2010 was also an uncontrolled practice-based trial of modified PIT, however, this study treated PNES only. No measures were collected at treatment end, but outcomes at an average of 42 months showed significant benefit in all domains measured except for economic activity. Effect sizes could not be calculated due to the nonnormal distribution of the measures reported. The quality of this 


\begin{tabular}{|c|c|c|c|c|c|c|c|}
\hline \multirow[b]{2}{*}{ Domain } & \multirow[b]{2}{*}{ Study } & \multirow[b]{2}{*}{ Measure } & \multirow[b]{2}{*}{ Population } & \multirow[b]{2}{*}{ Sample } & \multicolumn{3}{|c|}{ Effect sizes (pre-post) } \\
\hline & & & & & Treatment end & 6-month follow-up & $\begin{array}{l}\text { 12-month } \\
\text { follow-up }\end{array}$ \\
\hline \multirow[t]{6}{*}{ Physical symptoms } & Reuber $2007^{26}$ & PHQ-15 & All FND & 91 & 0.12 & 0.17 & \\
\hline & Hinson $2006^{25}$ & PMDRS & Motor FND & 10 & 1.26 & & \\
\hline & Mayor $2010^{27}$ & PHQ-15 & PNES & 47 & & & NC \\
\hline & & Seizure freq. & & & & & NC \\
\hline & Mayor $2010^{27}$ & SF-36 (P) & & & & & NC \\
\hline & Hubschmid $2015^{29}$ & SDQ-20 & All FND & 11 & $0.84^{*}$ & $1.07^{*}$ & * \\
\hline \multirow[t]{12}{*}{ Mental health } & Reuber $2007^{26}$ & CORE & All FND & 91 & 0.27 & 0.31 & \\
\hline & Russell $2016^{30}$ & BSI & PNES & 28 & 0.69 & & \\
\hline & Hinson $2006^{25}$ & Ham-D & Motor FND & 10 & 2.08 & & \\
\hline & & $\mathrm{BAI}$ & & & 1.99 & & \\
\hline & Mayor $2010^{27}$ & CORE & PNES & 47 & & & NC \\
\hline & & SF-36 (M) & & & & & NC \\
\hline & Kompoliti $2014^{28}$ & Ham-D & Motor FND & 7 & 0.51 & 0.57 & \\
\hline & & BAI & & & 0.70 & 1.52 & \\
\hline & Hubschmid $2015^{29}$ & CGI & All FND & 11 & $-0.16^{*}$ & $0.22^{*}$ & $0.73^{*}$ \\
\hline & & $\mathrm{BDI}$ & & & $0.54^{*}$ & $0.06^{*}$ & $0.28^{*}$ \\
\hline & & MADRS & & & $-0.24^{*}$ & $-0.19^{*}$ & $0.14^{*}$ \\
\hline & & SF-36 (M) & & & 0.83 & 1.35 & 2.09 \\
\hline \multirow[t]{2}{*}{ Well-being } & Reuber $2007^{26}$ & SF-36 & All FND & 91 & 0.32 & 0.41 & \\
\hline & Hubschmid $2015^{29}$ & SF-36 & All FND & 11 & 0.53 & -1.37 & 1.04 \\
\hline \multirow[t]{5}{*}{ Function } & Hinson $2006^{25}$ & PMDRS (F) & Motor FND & 10 & 1.08 & & \\
\hline & & GAF & & & 0.91 & & \\
\hline & Mayor $2010^{27}$ & Employment & PNES & 47 & & & NC \\
\hline & Hubschmid $2015^{29}$ & Rankin & All FND & 11 & $0.69^{*}$ & $1.16^{*}$ & $0.97^{*}$ \\
\hline & & Employment & & & 0.24 & 0.82 & 2.71 \\
\hline \multirow[t]{8}{*}{ Resource use } & Mayor $2010^{27}$ & Healthcare use & PNES & 47 & & & NC \\
\hline & Hubschmid $2015^{29}$ & Hospital days & All FND & 11 & -1.68 & * & * \\
\hline & & A\&E use & & & 1.56 & * & * \\
\hline & Russell $2016^{30}$ & Doctor visits & PNES & 28 & & & 0.32 \\
\hline & & Doctor cost & & & & & 0.35 \\
\hline & & Hospital visits & & & & & 0.55 \\
\hline & & Hospital days & & & & & 0.42 \\
\hline & & Hospital cost & & & & & 0.42 \\
\hline
\end{tabular}

${ }^{*}$ Calculated using OR.

A\&E, Accident and Emergency; BAI, Beck Anxiety Inventory; BDI, Beck Depression Inventory; BSI, Brief Symptom Inventory; CGI, Clinical Global Impression; CORE, Clinical Outcomes in Routine Evaluation; FND, functional neurological disorder; GAF, Global Assessment of Function; Ham-D, Hamilton Depression Rating Scale; MADRS, Montgomery-Asberg Depression Rating Scale; NC, not calculable; PDT, psychodynamic therapy; PHQ-15, 15-item Patient Health Questionnaire; PMDRS, Psychogenic Movement Disorders Rating Scale (F=function subscore); PNES, psychogenic nonepileptic seizures; SDQ-20, 20-item Strengths and Difficulties Questionnaire; SF36, 36-item Short Form health survey (M=mental health subscore/P=physical subscore).

study was rated as poor due to selection bias from participants self-selecting for treatment, and reporting bias from only 47 of 66 providing follow-up data from a mailed-out questionnaire.

Across PDT studies, all calculable pre-post (within-group) effect sizes were positive at the final time point, and generally moderate to large in size although there were time points at which the effect varied substantially in Hubschmid 2015. At treatment end, the median pooled pre-post effect size for PDT was 0.69 with a range from -1.68 to 2.08 , and at the final follow-up (excluding treatment end) it was 0.49 with a range from 0.14 to 2.71 .

There were only two controlled studies of PDT, both small. Kompoliti 2014 reported on a randomised delayed treatment crossover study of short-term dynamic psychotherapy for motor FND. Although beneficial effects were found in all measures when compared before and after, and between groups, the authors reported that the benefit was associated with time rather than treatment assignment. The small sample size, high dropout rate, potential for selection bias and lack of intention-to-treat analysis led to a rating of poor quality and mean that firm conclusions cannot be drawn from the data. Hubschmid 2015 reported statistically significant improvements following PIT for all FND in the majority of measures of physical and psychiatric symptoms, as well as well-being and some measures of healthcare use and no significant harms. All final pre-post (within group) effect sizes were positive and large (except Montgomery-Asberg Depression Rating Scale and Beck Depression Inventory which were small), however while most final effect sizes were positive or neutral when calculated between groups, two showed large negative effects (36-item Short Form health survey and Rankin). Closer examination reveals that, while randomisation was carried out, there were worse scores in the intervention group at baseline across every measure (except Clinical Global Impression) that were not taken into account. Coupled with the small sample size this precludes meaningful interpretation of betweengroup effect sizes in this study. At treatment end, the median pooled between group effect size for PDT was -0.03 with a range from -0.99 to 3.76 and at the final follow-up (excluding treatment end) it was 0.11 with a range from -1.28 to 0.98 . Non-numerical effect sizes were excluded. 


\section{Table 3 Effect sizes for pre-post comparisons in CBT studies}

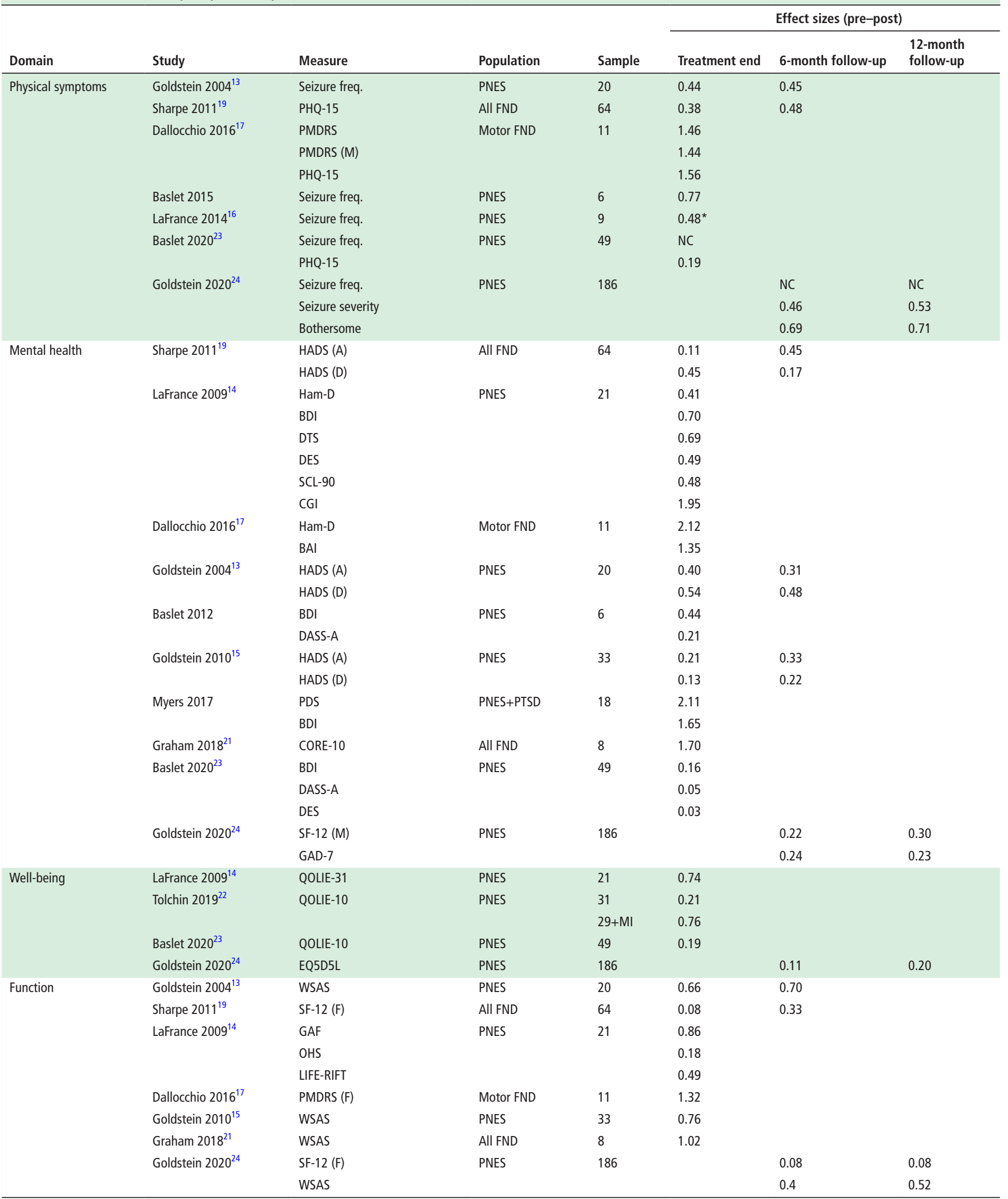

${ }^{*}$ Calculated using OR.

BAI, Beck Anxiety Inventory; BDI, Beck Depression Inventory; CBT, cognitive behavioural therapy; CGI, Clinical Global Impression; CORE-10, 10-item Clinical Outcomes in Routine Evaluation; DASS-A, Depression Anxiety Stress Scale anxiety subscore; DES, Dissociative Experiences Scale; DTS, Davidson Trauma Scale; EQ5D5L, EuroQol 5 Dimension 5-Level Scale; FND, functional neurological disorder; GAD-7, 7-item Generalised Anxiety Disorder Scale; GAF, Global Assessment of Function; HADS, Hamilton Anxiety and Depression Score (A=anxiety subscore/D=depression subscore); Ham-D, Hamilton Depression Rating Scale; LIFE-RIFT, Longitudinal Interval Follow-up Evaluation-Range of Impaired Functioning Tool; MI, motivational interviewing; NC, not calculable; OHS, Oxford Handicap Scale; PDS, Post-traumatic Diagnosis Scale; PHQ-15, 15-item Patient Health Questionnaire; PMDRS, Psychogenic Movement Disorders Rating Scale (M=motor subscore/ F=function subscore); PNES, psychogenic non-epileptic seizures; PTSD, post-traumatic stress disorder; QOLIE-31/10, 31/10-item Quality of Life in Epilepsy score; SCL-90, 90-item Symptom Checklist; SF-12, 12-item Short Form health survey ( $M=$ mental health subscore/F=function subscore); WSAS, Work and Social Adjustment Scale. 
Table 4 Effect sizes for comparisons of PDT with control and CBT with control

\begin{tabular}{|c|c|c|c|c|c|c|c|c|c|}
\hline \multirow[b]{2}{*}{ Domain } & \multirow[b]{2}{*}{ Study } & \multirow[b]{2}{*}{ Measure } & \multirow[b]{2}{*}{ Population } & \multirow[b]{2}{*}{ Sample } & \multirow[b]{2}{*}{ Control } & \multirow[b]{2}{*}{ Type } & \multicolumn{3}{|c|}{ Effect sizes (compared with control) } \\
\hline & & & & & & & Treatment end & $\begin{array}{l}\text { 6-month } \\
\text { follow-up }\end{array}$ & $\begin{array}{l}\text { 12-month } \\
\text { follow-up }\end{array}$ \\
\hline \multirow{9}{*}{$\begin{array}{l}\text { Physical } \\
\text { symptoms }\end{array}$} & Hubschmid $2015^{29}$ & SDQ-20 & All FND & 11 & $12 \mathrm{SC}$ & PDT & $0.84^{*}$ & $0.38^{*}$ & * \\
\hline & Sharpe $2011^{19}$ & PHQ-15 & All FND & 64 & $63 \mathrm{SC}$ & CBT & 0.25 & 0.13 & \\
\hline & Dallocchio $2016^{17}$ & PMDRS & Motor FND & 11 & $8 \mathrm{SC}$ & CBT & 1.41 & & \\
\hline & & PMDRS (M) & & & & & 1.99 & & \\
\hline & & PHQ-15 & & & & & 1.83 & & \\
\hline & Goldstein $2010^{15}$ & Seizure freq. & PNES & 33 & 33 SMC & CBT & $0.75 t$ & $0.42 \dagger$ & \\
\hline & Goldstein $2020^{24}$ & Seizure freq. & PNES & 186 & 182 SMC & CBT & & NC & NC \\
\hline & & Seizure severity & & & & & & 0.29 & 0.17 \\
\hline & & Bothersome & & & & & & 0.39 & 0.34 \\
\hline \multirow[t]{14}{*}{ Mental health } & Hubschmid $2015^{29}$ & BDI & All FND & 11 & $12 \mathrm{SC}$ & PDT & $0^{*}$ & $-0.52^{*}$ & 0 * \\
\hline & & MADRS & & & & & $-0.09 *$ & -0.41 * & 0 * \\
\hline & & SF-36 (M) & & & & & -0.05 & 0.24 & 0.98 \\
\hline & & CGI & & & & & $-0.32^{*}$ & $-0.12^{*}$ & $0.22^{*}$ \\
\hline & Kompoliti $2014^{28}$ & Ham-D & Motor FND & 7 & $8 \mathrm{SC}$ & PDT & 0.59 & & \\
\hline & & BAI & & & & & 0.53 & & \\
\hline & Dallocchio $2016^{17}$ & Ham-D & Motor FND & 11 & $8 \mathrm{SC}$ & CBT & 1.58 & & \\
\hline & & $\mathrm{BAl}$ & & & & & 1.31 & & \\
\hline & Sharpe $2011^{19}$ & $\operatorname{HADS}(\mathrm{A})$ & All FND & 64 & $63 \mathrm{SC}$ & CBT & 0.36 & 0.36 & \\
\hline & & HADS (D) & & & & & 0.29 & 0.32 & \\
\hline & Goldstein $2010^{15}$ & $\operatorname{HADS}(\mathrm{A})$ & PNES & 33 & 33 SMC & CBT & 0.20 & 0.20 & \\
\hline & & HADS (D) & & & & & 0.19 & 0.32 & \\
\hline & Goldstein $2020^{24}$ & SF-12 (M) & PNES & 186 & 182 SMC & CBT & & 0.32 & 0.16 \\
\hline & & GAD-7 & & & & & & 0.38 & 0.18 \\
\hline \multirow[t]{2}{*}{ Well-being } & Hubschmid $2015^{29}$ & SF-36 & All FND & 11 & $12 \mathrm{SC}$ & PDT & -0.92 & -2.22 & -1.28 \\
\hline & Goldstein $2020^{24}$ & EQ5D5L & PNES & 186 & 182 & CBT & & 0.33 & 0.33 \\
\hline \multirow[t]{7}{*}{ Function } & Sharpe $2011^{19}$ & SF-12 (F) & All FND & 64 & $63 \mathrm{SC}$ & CBT & 0.25 & 0.47 & \\
\hline & Hubschmid $2015^{29}$ & Rankin & All FND & 11 & $12 \mathrm{SC}$ & PDT & $-0.09^{*}$ & 0.31 * & $-0.57^{*}$ \\
\hline & & Employment & & & & & $3.76^{*}$ & $1.74^{*}$ & $0.26^{*}$ \\
\hline & Dallocchio $2016^{17}$ & PMDRS (F) & Motor FND & 11 & $8 \mathrm{SC}$ & CBT & 1.44 & & \\
\hline & Goldstein $2010^{15}$ & WSAS & PNES & 33 & 33 SMC & CBT & 0.59 & 0.64 & \\
\hline & Goldstein $2020^{24}$ & SF-12 (F) & PNES & 186 & 182 & CBT & & 0.22 & 0.27 \\
\hline & & WSAS & & & & & & 0.39 & 0.36 \\
\hline \multirow[t]{2}{*}{ Resource use } & Hubschmid $2015^{29}$ & Hospital days & All FND & 11 & $12 \mathrm{SC}$ & PDT & -0.99 & * & * \\
\hline & & A\&E use & & & & & 0.41 & * & * \\
\hline
\end{tabular}

${ }^{*}$ Calculated using OR.

†Effect size provided by authors.

A\&E, Accident and Emergency; BAI, Beck Anxiety Inventory; BDI, Beck Depression Inventory; CBT, cognitive behavioural therapy; CGI, Clinical Global Impression; EQ5D5L, EuroQol 5 Dimension

5-Level Scale; FND, functional neurological disorder; GAD-7, 7-item Generalised Anxiety Disorder Scale; HADS, Hamilton Anxiety and Depression Score (A=anxiety subscore/D=depression subscore); Ham-D, Hamilton Depression Rating Scale; MADRS, Montgomery-Asberg Depression Rating Scale; NC, not calculable; PDT, psychodynamic therapy; PHQ-15, 15-item Patient Health Questionnaire; PMDRS, Psychogenic Movement Disorders Rating Scale ( $M=$ motor subscore/F=function subscore); PNES, psychogenic non-epileptic seizures; SC, standard care; SDQ-20, 20-item Strengths and Difficulties Questionnaire; SF-12/36, 12/36-item Short Form health survey (M=mental health subscore/F=function subscore); SMC, standardised medical care; WSAS, Work and Social Adjustment Scale.

Among the Twelve studies of CBT identified, five were controlled (Goldstein 2010 and 2020, Sharpe 2011, LaFrance 2014 and Dallochio 2016). Four of the twelve provided follow-up data after the end of treatment (Goldstein 2004; 2010 and 2020, and Sharpe 2011). Most (nine) treated only PNES (with one of these, Myers 2017, treating only PNES with comorbid posttraumatic stress disorder (PTSD)). Of the others, two treated all FND (Sharpe 2011 and Graham 2018) and one (Dallocchio 2016) treated only motor FND. Quality ratings were generally higher for the CBT studies, with only two being rated as poor quality, Baslet 2015 due to a lack of detail about enrolment, inadequate power and outcome measures which changed over the course of the study and Baslet 2020 due to self-selection, low rates of completion (55\%) and high loss to follow-up.

Although most had small samples sizes (median $n=29$; range 6-367) there were three with moderate-to-large sample sizes, two focusing on PNES (Tolchin 2019 and Goldstein 2020; $\mathrm{n}=60$ and 367, respectively) and one on all FND (Sharpe 2011; $\mathrm{n}=127)$.

The largest study (Goldstein 2020), the Cognitive Behavioural Therapy for Dissociative non-Epileptic Seizures (CODES) trial, found no significant benefit of CBT for PNES on the primary measure of seizure frequency compared with a standardised psychiatric consultation control condition. However, there were statistically significant benefits of CBT on longest period of seizure freedom, psychosocial functioning, self-rated and clinician-rated global change, and healthcare treatment satisfaction compared with the control condition. The primary outcome effect size was not able to be calculated due to the non-normal distribution of the measures used; among secondary measures of physical symptoms, however, beneficial effects were moderate to large when compared before and after, and small to moderate 
when comparing to controls in reported seizure severity and bothersomeness. In the domains of mental health, well-being and function, beneficial effects were small to moderate when compared before and after and between groups, however, between group differences reduced modestly with time.

The majority of CBT studies showed statistically significant benefits on measures of physical symptoms but were inconsistent on other measures. Effect sizes which were able to be calculated were mostly moderate to large for physical symptoms and small to moderate on measures of mental health, function and quality of life. At treatment end, the median pooled pre-post effect size for CBT was 0.49 with a range from 0.03 to 2.12 , and at the final follow-up (excluding treatment end) it was 0.33 with a range from 0.08 to 0.71 . At treatment end, the median pooled between group effect size for CBT was 0.67 with a range from 0.19 to 1.99 and at the final follow-up (excluding treatment end) it was 0.32 with a range from 0.13 to 0.64 .

Comparing the pre-post medians of the pooled effect sizes at treatment end and at follow-up, the median effect size of PDT is higher on both occasions at 0.69 and 0.49 , respectively, compared with those for CBT at 0.49 and 0.33 , respectively. However, when comparing treatment with control, the picture is very different with the pooled median effect sizes for PDT at treatment end and end of follow-up being much lower for PDT at -0.03 and 0.11 , respectively, compared with 0.67 and 0.32 , respectively. The exclusion of non-calculable and non-numerical results has a large impact on the between group median effect size for PDT, which limits the utility of this comparison.

\section{DISCUSSION}

This review identified seven studies of PDT and eleven studies of CBT for FND. The relative preponderance of CBT studies may be related to the empirical tradition within that literature and possibly the relative ease of studying CBT, which may be more easily manualised and taught than some forms of PDT. Prepost effect sizes suggest that people with FND report moderate improvements in their mental and physical health, well-being and functioning after both treatments, supporting their use in clinical practice. The majority of the studies identified had small numbers of participants and most were uncontrolled, such that spontaneous resolution, placebo/non-specific effects and regression to the mean cannot be excluded as explanations for the positive clinical outcomes. The lack of high-quality controlled studies of PDT in particular means that its efficacy compared with no treatment remains to be determined.

There have been more well-conducted controlled studies pointing to the efficacy of CBT with different types of FND, in particular PNES. The greater proportion of studies on PNES may be because PNES is relatively homogeneous and more diagnostically straightforward compared with other subtypes of FND. We have treated them as part of the broader group of FND in our analysis, though there is some debate as to whether PNES should be considered a separate condition. ${ }^{3}$ One reason for 'splitting' PNES off would be if treatment was different, ${ }^{38}$ which may be the case in some respects; psychotherapeutic interventions for inherently paroxysmal PNES may be more likely to focus on triggers, for example, rather than sustaining factors. In terms of efficacy, however, there is no reason to assume that psychotherapy would be more effective in any one subtype of FND (particularly if combined with physical therapies where indicated, such as in motor FND), and this review supports that assertion by demonstrating comparable outcomes across a range of subtypes.
The most recently published study, the multisite CODES trial, contributes enormously to the quality of the evidence for CBT for PNES due to its size and robust design. On the one hand, this allows for a more confident assessment of the value of this modality for this subset of patients, which may be generalisable to other subtypes of FND to an extent; on the other hand, it allows for a more confident assessment of the limitations of CBT for PNES. Indeed, results suggest that the PNES-specific CBT intervention used was no more effective at reducing seizure frequency than standard medical care, with only a minority of participants being seizure free at 12 months (20\% with CBT vs $12 \%$ of controls); measures of anxiety and depression were also not significantly different between groups. The most important positive outcome was that psychosocial functioning was improved significantly, perhaps reflecting the underlying theoretical model and the focus on avoidance behaviour in the treatment. It may be that a CBT intervention specifically targeting distress and wider mental health issues (eg, PTSD) would be more effective. Heterogeneity within the patient group may also have contributed to the relatively modest effects observed in this study.

The lower quality of evidence for PDT for FND does not necessarily equate to the inferiority of this modality, and may reflect the relative immaturity of the treatment literature in that area. There is a clear need for more treatment research on PDT, particularly in light of the inconclusive findings of the CODES trial. In the absence of trials comparing the two approaches it is impossible to say which is the most effective treatment for FND; moreover, research on psychotherapies for other mental health conditions suggests that there is greater merit in studying which patients should receive which treatment rather than attempting to identify gross differences in overall treatment efficacy.

\section{Applying this review to clinical practice}

This review supports the ongoing use of psychotherapy for FND in clinical practice.

Both CBT and PDT are dependent on the patient accepting the possibility of psychological factors having an impact on their symptoms in order to engage in treatment. Some types of PDT (particularly psychoanalysis) take many years and require prolonged training and supervision. This is not true for some contemporary PDTs but, taken together, PDT is probably more expensive than CBT.

Because PDT involves identifying emotional and interpersonal patterns, patients need to be willing to explore these with the therapist. Some patients with FND initially attribute their symptoms to non-psychological causes, which may make it harder to address difficult underlying emotions. The association with alexithymia and dissociation as well as frequent mental health stigma and experiences of feeling dismissed by physicians among patients with FND may all contribute to increased resistance to this task, limiting the acceptability of PDT for some patients.

\section{Future directions}

An important target for future research is identifying patient characteristics that might predict better responses to psychotherapy or that might guide selection of a particular treatment modality in order to improve patient outcomes. For CBT, this might include maladaptive beliefs and behaviours (eg, activity avoidance) that are thought to be maintaining symptoms; for PDT, it may be emotional avoidance or maladaptive interpersonal patterns, including those that arise in relationships with doctors and other care providers. The high rates of personality 
vulnerability suggested by the standardised assessment of personality scores in the CODES trial may represent such a marker for the benefit of PDT and this may even go some way towards explaining why this treatment was less effective than anticipated in this population. Alternately, CBT may be an appropriate first step for all patients with FND, and those who fail to respond may then need the more interpersonally focused work that characterises PDT.

\section{LIMITATIONS}

This literature review is subject to a number of limitations, including the exclusion of unpublished studies and those published in languages other than English. The decision to limit the search to research published since the release of DSM-III in 1980 may also mean that potentially relevant studies were excluded; this was considered necessary, however, to ensure that samples resembled the current diagnostic entity of FND. Group therapy and inpatient psychotherapy were also excluded in order to narrow the focus of this review, however both are potentially beneficial for this population.

This systematic review calculated and compared effect sizes without distinction between primary and secondary study endpoints, which would normally be given different weighting. We feel this was justified as it allowed us to compare the outcomes of studies which would not otherwise be able to be compared but it does introduce a potential source of error.

Ideally this review would allow conclusions to be drawn about the differential benefit of each of the different modalities of CBT and PDT on each of the subtypes of FND; unfortunately, however, the variable quality of the different studies means that such a comparison is unlikely to be meaningful.

\section{CONCLUSIONS}

This review suggests that CBT and PDT have potentially positive effects, but the effects are not always seen in primary reduction of functional neurological symptoms. More high-quality treatment research is needed to confirm the efficacy of PDT in particular.

\section{Twitter Myles Gutkin @mjgutkin}

Acknowledgements MG would like to acknowledge Ms Nicola Wormleaton, librarian at Royal North Shore Hospital, for her assistance with search strategy design.

Contributors MG planned the review, carried out the analysis, wrote the first draft and modified all subsequent drafts. LM contributed to all drafts of the manuscript. $\mathrm{RB}$ contributed to the interpretation of the data and to all drafts of the manuscript. RK contributed to the interpretation of the data and to all drafts of the manuscript.

Funding The authors have not declared a specific grant for this research from any funding agency in the public, commercial or not-for-profit sectors.

Competing interests None declared.

Patient consent for publication Not required.

Provenance and peer review Not commissioned; externally peer reviewed.

Supplemental material This content has been supplied by the author(s). It has not been vetted by BMJ Publishing Group Limited (BMJ) and may not have been peer-reviewed. Any opinions or recommendations discussed are solely those of the author(s) and are not endorsed by BMJ. BMJ disclaims all liability and responsibility arising from any reliance placed on the content. Where the content includes any translated material, BMJ does not warrant the accuracy and reliability of the translations (including but not limited to local regulations, clinical guidelines, terminology, drug names and drug dosages), and is not responsible for any error and/or omissions arising from translation and adaptation or otherwise.

\section{ORCID iDs}

Myles Gutkin http://orcid.org/0000-0003-1700-7172

Loyola McLean http://orcid.org/0000-0002-6004-0093

Richard Brown http://orcid.org/0000-0003-3961-2023

Richard A Kanaan http://orcid.org/0000-0003-0992-1917

\section{REFERENCES}

1 Stone J, Carson A, Duncan R, et al. Who is referred to neurology clinics?--the diagnoses made in 3781 new patients. Clin Neurol Neurosurg 2010;112:747-51.

2 Carson A, Stone J, Hibberd C, et al. Disability, distress and unemployment in neurology outpatients with symptoms 'unexplained by organic disease'. J Neurol Neurosurg Psychiatry 2011;82:810-3.

3 Kanaan RAA, Duncan R, Goldstein LH, et al. Are psychogenic non-epileptic seizures just another symptom of conversion disorder? I Neurol Neurosurg Psychiatry 2017:88:425-9.

4 Edwards MJ, Adams RA, Brown H, et al. A Bayesian account of 'hysteria'. Brain 2012;135:3495-512.

5 Pick S, Goldstein LH, Perez DL, et al. Emotional processing in functional neurological disorder: a review, biopsychosocial model and research agenda. J Neurol Neurosurg Psychiatry 2019;90:704-11.

6 Brown RJ, Reuber M. Towards an integrative theory of psychogenic non-epileptic seizures (PNES). Clin Psychol Rev 2016;47:55-70.

7 Blagys MD, Hilsenroth MJ. Distinctive features of short-term psychodynamicinterpersonal psychotherapy: a review of the comparative psychotherapy process literature. Clin Psychol 2000;7:167-88.

8 Brown P, Macmillan MB, Meares R, et al. Janet and Freud: revealing the roots of dynamic psychiatry. Aust N Z J Psychiatry 1996;30:480-9.

9 Beck AT. Cognitive therapy: nature and relation to behavior therapy. Behav Ther 1970;1:184-200.

10 Monzoni CM, Duncan R, Grünewald R, et al. Are there interactional reasons why doctors may find it hard to tell patients that their physical symptoms may have emotional causes? A conversation analytic study in neurology outpatients. Patient Educ Couns 2011;85:e189-200.

11 Ruddy R, House A. Psychosocial interventions for conversion disorder. Cochrane Database Syst Rev 2005;4:CD005331.

12 Carlson P, Nicholson Perry K. Psychological interventions for psychogenic non-epileptic seizures: a meta-analysis. Seizure 2017;45:142-50.

13 Goldstein LH, Deale AC, Mitchell-O'Malley SJ, et al. An evaluation of cognitive behavioral therapy as a treatment for dissociative seizures: a pilot study. Cogn Behav Neurol 2004;17:41-9.

14 LaFrance WC, Miller IW, Ryan CE, et al. Cognitive behavioral therapy for psychogenic nonepileptic seizures. Epilepsy Behav 2009;14:591-6.

15 Goldstein LH, Chalder T, Chigwedere C, et al. Cognitive-behavioral therapy for psychogenic nonepileptic seizures: a pilot RCT. Neurology 2010;74:1986-94.

16 LaFrance WC, Baird GL, Barry JJ, et al. Multicenter pilot treatment trial for psychogenic nonepileptic seizures: a randomized clinical trial. JAMA Psychiatry 2014;71:997-1005.

17 Dallocchio C, Tinazzi M, Bombieri F, et al. Cognitive behavioural therapy and adjunctive physical activity for functional movement disorders (conversion disorder): a pilot, single-blinded, randomized study. Psychother Psychosom 2016;85:381-3.

18 Baslet G, Dworetzky B, Perez DL, et al. Treatment of psychogenic nonepileptic seizures: updated review and findings from a mindfulness-based intervention case series. Clin EEG Neurosci 2015;46:54-64.

19 Sharpe M, Walker J, Williams C, et al. Guided self-help for functional (psychogenic) symptoms: a randomized controlled efficacy trial. Neurology 2011;77:564-72

20 Myers L, Vaidya-Mathur U, Lancman M. Prolonged exposure therapy for the treatment of patients diagnosed with psychogenic non-epileptic seizures (PNES) and posttraumatic stress disorder (PTSD). Epilepsy Behav 2017:66:86-92.

21 Graham CD, O'Hara DJ, Kemp S. A case series of acceptance and commitment therapy (act) for reducing symptom interference in functional neurological disorders. Clin Psychol Psychother 2018;25:489-96.

22 Tolchin B, Baslet G, Suzuki J, et al. Randomized controlled trial of motivational interviewing for psychogenic nonepileptic seizures. Epilepsia 2019;60:986-95.

23 Baslet G, Ehlert A, Oser M, et al. Mindfulness-based therapy for psychogenic nonepileptic seizures. Epilepsy Behav 2020;103:106534.

24 Goldstein LH, Robinson EJ, Mellers JDC, et al. Cognitive behavioural therapy for adults with dissociative seizures (codes): a pragmatic, multicentre, randomised controlled trial. Lancet Psychiatry 2020;7:491-505.

25 Hinson VK, Weinstein S, Bernard B, et al. Single-blind clinical trial of psychotherapy for treatment of psychogenic movement disorders. Parkinsonism Relat Disord 2006; 12:177-80

26 Reuber M, Burness C, Howlett S, et al. Tailored psychotherapy for patients with functional neurological symptoms: a pilot study. J Psychosom Res 2007:63:625-32.

27 Mayor R, Howlett S, Grünewald R, et al. Long-term outcome of brief augmented psychodynamic interpersonal therapy for psychogenic nonepileptic seizures: seizure control and health care utilization. Epilepsia 2010;51:1169-76.

28 Kompoliti K, Wilson B, Stebbins G, et al. Immediate vs. delayed treatment of psychogenic movement disorders with short term psychodynamic psychotherapy: randomized clinical trial. Parkinsonism Relat Disord 2014;20:60-3. 
29 Hubschmid M, Aybek S, Maccaferri GE, et al. Efficacy of brief interdisciplinary psychotherapeutic intervention for motor conversion disorder and nonepileptic attacks. Gen Hosp Psychiatry 2015;37:448-55.

30 Russell LA, Abbass AA, Allder SJ, et al. A pilot study of reduction in healthcare costs following the application of intensive short-term dynamic psychotherapy for psychogenic nonepileptic seizures. Epilepsy Behav 2016;63:17-19.

31 Santos NdeO, Benute GRG, Santiago A, et al. Psychogenic non-epileptic seizures and psychoanalytical treatment: results. Rev Assoc Med Bras 2014:60:577-84.

32 American Psychological Association. Fear reduction and fear behavior: problems in treating a construct. Research in psychotherapy conference; 3rd, May-Jun, Chicago, IL, US, 1966.
33 Williams C, Kent C, Smith S, et al. Overcoming functional neurological symptoms: a five areas approach. Abingdon, United Kingdom: Hodder Arnold; United Kingdom, 2011.

34 Foa EB, Hembree EA, Rothbaum BO. Prolonged exposure therapy for PTSD : emotional processing of traumatic experiences : therapist guide. Oxford ; New York: Oxford University Press, 2007.

35 Davanloo H. Short-term dynamic psychotherapy. Northvale, NJ: Aronson, 1980.

36 Guthrie E. Psychodynamic interpersonal therapy. Adv Psychiatr Treat 1999:5:135-45.

37 Breuer J, Freud S. Studies in Hysteria. In: Freud S, Strachey J, Freud A, eds. The standard edition of the complete psychological works of Sigmund Freud. London: Hogarth Press, 1895.

38 Wessely S, Nimnuan C, Sharpe M. Functional somatic syndromes: one or many? Lancet 1999;354:936-9. 